\title{
Ultrasound Curricula of Student Education in Europe: Summary of the Experience
}

(c) (1) $\odot$

Authors

Helmut Prosch ${ }^{(\mathbb{D})}$, Maija Radzina2,3, Christoph F. Dietrich ${ }^{(\mathbb{D}}$, Michael Bachmann Nielsen 5,6 , Sven Baumann?, Caroline Ewertsen ${ }^{5}$, Christian Jenssen ${ }^{8}$, Adnan Kabaalioğlu9 ${ }^{9}$, Wojciech Kosiak ${ }^{10}$, Wolfgang Kratzer ${ }^{7}$, Adrian Lim ${ }^{11}$, Alina Popescu ${ }^{12}$, Vladimir Mitkov ${ }^{13}$, Cosima Schiavone ${ }^{14}$, Martin Wohlin ${ }^{15}$, Matthias Wüstner ${ }^{16}$, Vito Cantisani ${ }^{17}$

Affiliations

1 Department of Biomedical Imaging and Image-Guided Therapy, Medical University of Vienna, Vienna General Hospital, Vienna, Austria

2 Riga Stradins University, Radiology department, Riga, Latvia

3 University of Latvia, Medical Faculty, Paula Stradina clinical university hospital, Diagnostic Radiology Institute Riga, Latvia

4 Department Allgemeine Innere Medizin, Kliniken Hirslanden Beau Site, Salem und Permanence, Bern, Switzerland

5 Department of Radiology, Rigshospitalet, Copenhagen, Denmark

6 Department of Clinical Medicine, University of Copenhagen, Copenhagen, Denmark

7 Department of Internal Medicine I, Ulm University Hospital, Ulm, Germany

8 Department of Internal Medicine, Krankenhaus Märkisch-Oderland, Strausberg, and Brandenburg Institute for Clinical Ultrasound, Neuruppin, Germany

9 Department of Radiology, Koç University Hospital-istanbul, Turkey

10 Department of Paediatrics, Haematology and Oncology, Medical University of Gdansk, Poland

11 Department of Imaging, Imperial College London and Healthcare Trust, UK

12 Department of Gastroenterology, "Victor Babeș" University of Medicine and Pharmacy Timișoara, Timișoara, Romania

13 Diagnostic Ultrasound Department, Russian Medical Academy of Continuous Professional Education, Moscow, Russian Federation)

14 Unit of Internistic Ultrasound, Department of Medicine and Science of Aging, „G. d'Annunzio“ University, Chieti, Italy

15 Department of Medical Sciences, Uppsala Universitet, Uppsala, Sweden

16 Central Interdisciplinary Sonography, Krankenhaus der Barmherzigen Brüder Trier, Germany

17 Department of Radiological, Oncologic and Anatomo-pathologic Sciences, University of Rome La Sapienza, Roma, Italy

Key words

ultrasound, methods \& techniques, guideline, teaching

$\begin{array}{ll}\text { received } & 03.01 .2020 \\ \text { revised } & 11.05 .2020 \\ \text { accepted } & 13.05 .2020\end{array}$

Bibliography

Ultrasound Int Open 2020; 6: E25-E33

DOI 10.1055/a-1183-3009

ISSN 2199-7152

(c) 2020. The Author(s).

This is an open access article published by Thieme under the terms of the Creative Commons Attribution-NonDerivative-NonCommercial-License, permitting copying and reproduction so long as the original work is given appropriate credit. Contents may not be used for commecial purposes, or adapted, remixed, transformed or built upon. (https://creativecommons.org/ licenses/by-nc-nd/4.0/)

Correspondence:

Dr. Christoph F. Dietrich, MBA

Kliniken Hirslanden Beau Site, Salem und Permanence,

Department Allgemeine Innere Medizin

Schänzlihalde

3036 Bern

Switzerland

Tel.: +41798347180, Fax : +41798347180

christophfrank.dietrich@hirslanden.ch

\section{ABSTRACT}

Background Despite the increasing role of ultrasound, structured ultrasound teaching is only slowly being integrated into the curricula of medical schools and universities all over Europe. Aim To survey the current situation at European universities regarding the integration of ultrasound in student medical education and to report on models of student ultrasound training from selected European universities.

Methods A questionnaire survey focusing on the implementation of curricular ultrasound education was sent out to the 28 presidents of the national ultrasound societies of the European Federation of Societies for Ultrasound in Medicine and Biology (EFSUMB), who were asked to distribute the questionnaires to the medical universities of their countries.

Results Overall, 53 questionnaires were returned from 46 universities in 17 European countries. In most of the universities (40/46 universities, $87 \%$ ), the theoretical background of ultrasound is taught. However, in only a minority of universities is 
ultrasound integrated in anatomy courses (8/46 universities, $17 \%$ ) or basic science courses (16/46 universities, 35\%). Practical skills in ultrasound are taught in $56 \%$ of the universities (26/46 universities) and tested in a practical exam in seven of the responding universities (15\%). The number of hours in which ultrasound was taught ranged from one to 58 (mean, seven). The respondents reported that lack of time and limited faculty funding were major hurdles.
Conclusion According to our survey, only a minority of European universities has integrated ultrasound into the preclinical curriculum thus far. Future EFSUMB initiatives will continue to promote the introduction of ultrasound as an integrative part of the core curriculum of student medical education, and the preparation of proper teaching material.

\section{Introduction}

In the last decade, ultrasound has become an indispensable diagnostic tool in almost every medical specialty. As a consequence, in many European countries, ultrasound has been integrated into the training curricula of resident programs in many countries. However, despite the increasing role of ultrasound, structured ultrasound teaching is only slowly being integrated into the curricula of medical schools all over Europe.

Therefore, to promote the integration of ultrasound training into the curricula of universities, the European Federation of Societies for Ultrasound in Medicine and Biology (EFSUMB) has published policy statements on medical student education in ultrasound [1,2] and the World Federation for Ultrasound in Medicine and Biology (WFUMB) has published position papers [3]. In the EFSUMB statement paper, the authors suggest that student ultrasound training be integrated into both the preclinical and clinical curriculum. The statement paper further emphasizes the importance of sharing present knowledge regarding student education. However, as the curricula among universities are very heterogeneous, it is not feasible to devise one ultrasound curriculum that would fit into the diverse curricula of all universities. Consequently, individual universities have to develop their own ultrasound curricula that fit into their existing structures.

The aim of the present paper was to survey the current situation at European universities regarding the integration of ultrasound in student medical education and to discuss the implications. Furthermore, faculty members from several European universities were asked to share their experience regarding student ultrasound training.

\section{Materials and Methods}

\section{Data collection}

In June 2016, a questionnaire designed to survey the current situation at European universities regarding the integration of ultrasound in student medical education was sent to all 28 presidents of the ultrasound societies represented in EFSUMB. The presidents were asked to forward the questionnaires to the deans or representatives of national medical universities. The questionnaire was created using Microsoft Word 2016 (Word 2016, Microsoft, Bellevue, WA, USA). The data could be entered in the respective fields of the form and sent back via e-mail or returned via fax or regular mail. The questionnaire surveyed how ultrasound is already imple- mented at universities throughout Europe. In reference to a similar survey undertaken in the USA [4], the respondents were asked to indicate why they integrated ultrasound into the curriculum, to explain the methods, the difficulties, and barriers, and to give suggestions for improvement. Following the survey, a selected number of faculty members from a variety of European countries were asked to summarize how ultrasound was integrated into the curriculum of their universities.

\section{Data handling and statistical analysis}

The data of the returned questionnaires were entered manually into a Microsoft Excel spreadsheet. Statistical analysis was performed using Microsoft Excel 2016 (Excel 2016, Microsoft, Bellevue, WA, USA). All data were described in absolute and relative frequencies. If more than one questionnaire was returned from one university, the responses were considered as one response if the answers were consistent. In case of discrepant answers from one university, answers were considered individually.

\section{Results}

\section{Survey}

Overall, 53 questionnaires were returned from 46 universities in 17 European countries. At most of the universities (40/46 universities, $87 \%)$, the theoretical background of ultrasound is taught. However, ultrasound is integrated into anatomy courses (8/46 universities, $17 \%$ ) or basic science courses (16/46 universities, $35 \%$ ) at only a minority of universities. Practical skills in ultrasound are taught at $56 \%$ of the universities (26/46 universities) and tested in a practical exam at 7 of the responding universities (15\%). The number of lessons in which ultrasound was taught ranged from 1 to 58 (mean: 7). When asked about obstacles hindering the integration of ultrasound into the curricula, the most commonly reported obstacle was lack of time (27/44 respondents, $61 \%)$, followed by faculty funding (17/39, $43 \%)$, and equipment funding (18/40, $45 \%)$ ( $\triangleright$ Table 1$)$. When asked about the reasons for ultrasound implementation, $90 \%$ of all respondents (37/41) indicated that ultrasound knowledge is useful for all physicians, and an almost equal number of respondents (34/39, $87 \%$ ) were convinced that ultrasound knowledge was useful to learn later in practice. $60 \%$ of all respondents (23/38) indicated that ultrasound helps students learn anatomy ( $\triangleright$ Table 2$)$. 
- Table. 1 Percieved obstacles hindering the integration of ultrasound into the curricula

\begin{tabular}{|c|c|c|c|c|c|c|}
\hline & $\begin{array}{l}\text { Not } \\
\text { important }\end{array}$ & $\begin{array}{l}\text { Somewhat } \\
\text { important }\end{array}$ & Indifferent & Important & $\begin{array}{l}\text { Very } \\
\text { important }\end{array}$ & $\begin{array}{l}\text { Not } \\
\text { answered }\end{array}$ \\
\hline Lack of funding for faculty & 4 & 6 & 12 & 8 & 9 & 14 \\
\hline Lack of funding for equipment & 3 & 8 & 11 & 5 & 13 & 13 \\
\hline Lack of time in current medical curriculum & 7 & 8 & 4 & 12 & 15 & 7 \\
\hline Difficulty with faculty recruitment/training & 4 & 7 & 11 & 7 & 7 & 17 \\
\hline $\begin{array}{l}\text { Lack of acceptance or understanding of ultrasound } \\
\text { political barriers }\end{array}$ & 15 & 3 & 5 & 6 & 5 & 19 \\
\hline No barriers & 1 & 1 & 3 & 0 & 3 & 45 \\
\hline
\end{tabular}

- Table. 2 Reasons for ultrasound implementation

\begin{tabular}{|l|l|l|l|l|l|}
\hline & $\begin{array}{l}\text { Not } \\
\text { important }\end{array}$ & $\begin{array}{l}\text { Somewhat } \\
\text { important }\end{array}$ & $\begin{array}{l}\text { Indifferent } \\
\text { Important } \\
\text { important } \\
\text { answered }\end{array}$ \\
\hline Useful to learn later in practice & 0 & 4 & 1 & 13 & 21 \\
\hline Helps students learn anatomy & 4 & 1 & 10 & 5 & 18 \\
\hline Ultrasound and teaching are fun & 1 & 7 & 12 & 4 & 13 \\
\hline Benefits school's prestige & 8 & 3 & 8 & 7 & 11 \\
\hline Ultrasound knowledge is useful for all physicians & 0 & 1 & 3 & 4 & 33 \\
\hline
\end{tabular}

\section{Experience of European Universities}

Vienna, Austria

\section{Local structure}

At the Medical University of Vienna, 640 students enroll each year. The whole curriculum takes 6 years with 18 weeks of clerkship and a clinical internship of 48 weeks.

\section{Content of ultrasound lectures}

All students attend lectures on the physical background of ultrasound and its indications in the first semester and see anatomical as well as pathological US images in their anatomy classes.

\section{Who is teaching?}

Radiologists give the lectures on the physical background, as well as on ultrasound anatomy and pathology. In the hands-on course, one teacher trains each group of students, either a radiologist or a specially trained student tutor. The tutors are trained in an elective course and must have sufficient tutoring experience in peer teaching before being accepted as a tutor for the student course.

In every course, two groups of students are trained in one room with one radiologist and one student tutor teaching. In this manner, it can be ensured that more challenging questions that might be too difficult for the student tutor can also be answered.

\section{Hands-on courses}

Mandatory hands-on courses take place at the end of the $7^{\text {th }}$ semester and consist of $3 \times 2$ hours within three weeks. In this course, students are trained in groups of 5 students. 6 of the 14 ultrasound devices used for this course are routine US machines from the department of radiology, 4 US devices are from the students' skills lab, and 4 machines are rented from an ultrasound vendor each year for the period of the course.

\section{Examinations (learning success, testing)}

At the end of the $8^{\text {th }}$ semester, all students have to pass an objective, structured, clinical examination (OSCE). Of the 12 practical OSCE stations, one is on ultrasound, where each student has to show one out of six of the standard views and must answer US anatomy and pathology questions (i.e., cholecystolithiasis, cholecystitis, pleural and pericardial effusions, ascites, hydronephrosis).

\section{Riga, Latvia}

\section{Local structure}

There are two medical faculties in Latvia: The Riga Stradins University (RSU) medical faculty enrolls 250 students each year and the University of Latvia (LU) enrolls 70 students annually. Both universities provide mandatory formal teaching in medical ultrasound over the six years at RSU and over two years at LU.

\section{Content of lectures}

Students attend lectures about the basics of ultrasound, which are also available in student e-platform studies. Starting in 2013, RSU began to use the ultrasound simulator, ULTRASIM, in the field of 
abdominal, neck ultrasound, obstetrics, and gynecology. Students attend lectures about the basics of ultrasound for two hours, which are also available in student e-platform studies. Ultrasound is integrated into the basic studies for 15 hours at RSU. LU has integrated medical ultrasound into the diagnostic imaging course with lectures and practical sessions for up to six hours of training. There is one more optional practical and theoretical class about the basics of ultrasound held at the RSU Department of Radiology, with 12 students per one supervisor radiologist. Following the demonstration lesson, students can practice on the ultrasound simulator for 32 hours. Following the demonstration lesson, students can spend free time practicing on the ultrasound simulator.

\section{Who is teaching?}

The ultrasound lectures at RSU and LU are conducted by members of the interdisciplinary team, which consists of radiologists, cardiologists, anesthesiologists, and certified ultrasound specialists from various specialties, all with experience in the use of ultrasound and who have board certification. Radiology trainees supervise the practical courses. These trainees had successfully applied for a practical ultrasound teaching program at the University Hospital's ultrasound unit that lasted at least two years, in which they acquired deeper knowledge through the examination of patients on a daily basis and had proven their skills and knowledge after the exam.

\section{Hands-on courses}

At the beginning of the clinical section, all students in the fifth, or, optionally, the sixth semester, are offered a place in this elective, parallel to the clinical examination courses. The practical course of LU includes two two-hour sessions with groups consisting of ten students. The participants perform all the exercises on each other under the supervision of the tutor. Current high-end ultrasound equipment or a dedicated portable ultrasound machine from the university hospital's ultrasound unit for point-of-care ultrasound (POCUS) are used for training. The ULTRASIM simulator allows students to practice the performance of ultrasound examination on a mannequin while viewing real-time ultrasound images. Students fill out evaluation forms at the end of the ultrasound course. The second-level ultrasound program is implemented in the 5 th year radiology program, with one class dedicated to ultrasound pathology. Students have one hour of supervised hands-on training.

\section{Examinations}

At the end of the lecture series, there is a written multiple-choice test. The practical course finishes with a practical and oral examination based on a standardized assessment process. Successful attendance is obligatory for all students and is a prerequisite for further courses.

\section{Ulm, Germany}

\section{Local structure}

Currently, a total of 325 students begin their studies every year at the medical faculty of the University in Ulm. The entire curriculum requires six years and is divided into two years of preclinical studies and another four years of clinical education, including several clinical internships and three trimesters of a practical year. Ultra- sound has been a mandatory component of the human medicine degree curriculum since the winter term of 2016/17.

\section{Content of lectures}

The Ulm ultrasound course consists of lectures and a practical course conducted in small groups. The lecture-based part regularly includes an interdisciplinary approach (including cardiology, hepatology, gastroenterology, endocrinology, radiology, pediatrics, anesthesiology, and others). It conveys the theoretical content, basic principles, and potential application areas of ultrasound. The practical course imparts the basic handling of the equipment, as well as a fundamental understanding of anatomic structures. The course also includes instructions for examinations of the liver, gallbladder and bile ducts, kidneys and adrenal glands, spleen, pancreas, abdominal vessels, and lymph nodes.

\section{Who is teaching?}

The ultrasound lectures are conducted by members of the interdisciplinary ultrasound team, which consists of internists, cardiologists, radiologists, pediatricians, and anesthesiologists, all with experience in the use of ultrasound. The practical course is supervised by tutors who are medical students. All of them have attended the course themselves. They subsequently successfully applied for a practical ultrasound course that lasts at least four weeks at the university hospitals' interdisciplinary ultrasound unit, in which they acquired deeper knowledge through the examination of patients on a daily basis.

\section{Hands-on courses}

At the beginning of the clinical section, all students in the fifth or, optionally, the sixth semester are offered a place in an elective, parallel to the clinical examination courses. The lecture series consists of 13 units of 45-minute sessions. The practical course includes four two-hour sessions with groups that consist of four students. Within this group, the participants perform all the exercises on each other under the supervision of the tutor. All groups use current, high-end ultrasound equipment from the university hospital's interdisciplinary ultrasound unit. Both parts of the course require compulsory attendance.

\section{Examinations}

At the end of the lecture series, there is a written, multiple-choice test. The practical course finishes with a practical and oral examination based on a standardized assessment process. Successful attendance is obligatory for all students and is a prerequisite for further courses.

\section{Chieti, Italy}

\section{Local structure}

About 220 students begin each first year of the six-year course of medical school at “G. D’Annunzio" University of Chieti. Participation in this ultrasound course is mandatory.

\section{Content of lectures}

The university offers ultrasound training for $3^{\text {rd }}$ and $4^{\text {th }}$ year students. The theoretical part regularly involves multiple disciplines. It includes a review of basic principles and application areas of ultrasound. The practical course imparts the basic handling of the equipment, as well as a fundamental understanding of the anatom- 
ic structures on the organ systems of the abdomen, thorax, heart, lymphatic system, thyroid, and blood vessels.

\section{Who is teaching?}

The ultrasound courses are held by internists (professors, medical doctors, PhD students, and residents) with many years of ultrasound experience according to the guidelines of the Italian Society for Ultrasound in Medicine and Biology (SIUMB) and of EFSUMB. The sessions consist of a theoretical part on the basics of ultrasound and a more important practical section, which includes live demonstrations and hands-on training. During the lessons, there are often presentations of images and videos that demonstrate physiological and pathological ultrasound findings.

\section{Hands-on courses}

The courses are organized over four weeks, with practical training of two hours/week. The students are divided into groups of 5-10 students for each teacher and ultrasound machine for better understanding of ultrasound technique and to have a real possibility to experience "hands-on" ultrasound. The students are usually also the models for hands-on sessions.

\section{Examinations (learning success, testing)}

There is no written or multiple-choice test, but ultrasound skills are evaluated in a practical test at the end of the training period. All participants fill in a questionnaire to assess the satisfaction with the course. Medical students with a special interest in medical ultrasound are invited to participate in advanced lessons and courses organized by university or scientific societies.

\section{Gdansk, Poland}

\section{Local structure}

At the Medical University of Gdańsk, 300 students are accepted each year. The whole curriculum requires six years. Currently, there are no obligatory US courses. Interested students, however, are invited to participate in an optional abdominal and chest ultrasound course and/or join an ultrasound student scientific circle (about 350 members in 2019). There are plans to incorporate an ultrasound course into the curriculum in the future.

\section{Content of lectures}

The objectives of the voluntary ultrasound course are to obtain skills in handling ultrasound devices and to visualize abdominal organs and detect possible abnormalities. The course is divided into the following sections: 1 . Ultrasound handling; 2 . Gallbladder and biliary ducts; 3 . Vessels of the liver; 4. Liver segments; 5 . Pancreas and spleen; 6 . Kidneys and the urinary system; and 7 . Big vessels of the abdomen. Each section starts with a 20-minute demonstration for groups of 15-20 students led by experienced doctors. Afterward, the students have about two to four weeks to master each section by themselves. No simulators are used. Students practice on each other or on volunteers. Students practice at the Workshop of Diagnostic Ultrasound at the pediatric, hematologic, and oncologic wards at the Medical University of Gdansk, where well-trained doctors are available from Monday to Friday for six hours a day. In addition, students can repeat and deepen their knowledge using a Polish education website platform (www.eduson.pl), where each section is ex- plained in an easy and simple way, with images and movies that can be used as a reference (mainly while practicing on a machine).

On average, students spend $42 \mathrm{~h}$ and $24 \mathrm{~min}$ (minimal $16 \mathrm{~h}$ and maximal $70 \mathrm{~h}$ ) practicing by themselves during the whole course.

The ultrasound scientific club has no predefined content. There are a few theoretical and practical events every year with unlimited access to the ultrasound machines throughout the academic year and during the holidays.

\section{Who is teaching?}

Experienced doctors and a professor are available for the course, all of whom are pediatricians who perform ultrasound exclusively in everyday practice.

The same experienced specialists are available for the ultrasound scientific club, where older and more experienced students ('leaders') also help younger students when appointed by the professor and after passing an exam.

\section{Hands-on courses}

One machine is available for interested students (course or ultrasound scientific club) for practice by themselves all day (7:0022:00, seven days/week), two in the afternoon (14:00-22:00) and another two all day or in the afternoon only, depending on availability (may be used for patient examination if needed).

\section{Examinations (learning success, testing)}

After each chapter, there is a practical test, where each student is asked to visualize particular organs or anatomical structures, to perform particular tasks (measurements, different sections of organs, Doppler examinations), and to answer a few theoretical questions. No examinations are mandatory for the ultrasound scientific club, except for the 'leaders,' similar to the examinations in the course. The required knowledge includes ultrasound examinations of the whole abdomen, thyroid, and lungs.

\section{Timișoara, Romania}

\section{Local structure}

Approximately 600 students begin the courses at the "Victor Babeș" University of Medicine and Pharmacy Timișoara each year. The curriculum for general medicine requires six years, two years of pre-clinical and four years of clinical courses.

\section{Content of lectures}

Currently, the university curriculum offers a basic course in abdominal ultrasound for all students in the $3^{\text {rd }}$ year and an optional course in echocardiography and abdominal ultrasound for the students from the $4^{\text {th }}$ year (this one is attended by around 100 students every year), both structured as one hour/week courses and one hour/week practical sessions for 14 weeks (one semester). These courses include lectures on basic principles and ultrasound examination technique, normal abdominal ultrasound anatomy, and primary indications for abdominal ultrasound and ultrasound pathology related to the abdominal organs. There is also, on the university website, an e-Book of Ultrasound for Students in Romanian, English, and French, with text and pictures available for all students and also for external use (http://www.umft.ro/data_files/documente-atasate-sectiuni/2213/curs_20eco_20eng.pdf). 


\section{Who is teaching?}

Gastroenterologists, professors, associate professors and lecturers, who are experts (level III) in ultrasound of the Romanian Society of Ultrasound in Medicine and Biology (SRUMB) and EFSUMB hold the ultrasound courses. The practical sessions are based on live demonstrations, hands-on training, and video presentations, and are also held by gastroenterologists, assistant professors, and PhD students. Usually, one teacher trains two groups. A gallery of images and short movies of pathological conditions for students in training are under development. Sometimes, at the student's request, a two-day additional ultrasound course for students is arranged during the weekend, with short theoretical lectures (approximately two hours) and mainly hands-on activities with portable ultrasound machines. Postdoc and PhD students are primarily involved in these types of activities, but with the strong support of the professors and associate professors.

\section{Hands-on courses}

The courses are organized during one semester (14 weeks in length), with lectures for one hour/week and practical training for one hour/week. During the practical sessions, the students are divided into groups of 7-10 students, each group usually using one ultrasound machine (five machines are prepared for this purpose). The hands-on sessions are based on learning normal ultrasound anatomy using students as models. Teachers demonstrate pathological conditions.

\section{Examinations (learning success, testing)}

The evaluation period is at the end of the semester and consists of a theoretical multiple-choice test and a practical exam, in which students must recognize and describe frequent ultrasound pathologies from video cases. A questionnaire about their satisfaction regarding the ultrasound course is offered to students at the end of their activity and the results are evaluated at the university level. The best students from the ultrasound courses are invited to participate in advanced ultrasound training to become tutors for future ultrasound courses.

\section{Uppsala, Sweden}

\section{Local structure}

At the Medical School of Uppsala University, 250 students begin each year. The whole curriculum requires 5.5 years: 2 years pre-clinical courses and 3.5 years clinical courses. After graduation, a twoyear internship (Foundations Years) follows, which leads to board certification.

\section{Content of lectures}

During the $3^{\text {rd }}$ year of the medical program, the students begin to learn the basic principles of ultrasound. Ultrasound is integrated into the curriculum, and the FATE (Focused Assessed Transthoracic Echocardiography) protocol is used. As preparation for a half day (four hours) of knobology and practicing ultrasound, students go through e-learning (https://sonosim.ttlms.com/signon/). Teaching takes place in groups of four to seven students per facilitator. The facilitator is a junior doctor trained in ultrasound and the FATE protocol. During the last year, medical students also learn the
"FAST" (Focused Assessment with Sonography in Trauma) protocol, with basically the same setup as for the FATE protocol. Facilitators are residents in emergency medicine at the Uppsala University Hospital. The FAST protocol is integrated into the course of emergency medicine, taken just before graduation.

\section{Who is teaching?}

Specially trained junior doctors hold lectures on the physical science basis, as well as on ultrasound anatomy and pathology. In hands-on courses, a specially trained junior doctor trains each group of students. The junior doctors are well trained in the clinic and have sufficient tutoring experience before being accepted as a tutor for the course. In every course, small groups of students train in one room. In every room, there is one student tutor teaching.

\section{Hands-on courses}

Mandatory hands-on courses take place at the end of the $5^{\text {th }}$ semester. In this course, students are trained in groups of five students. 6 of the 14 ultrasound devices used for this course are handheld ultrasound devices and the rest of the devices are a mix of brand new and slightly older, less mobile ultrasound equipment.

\section{Examinations (learning success, testing)}

During year 3, all students go through e-learning (https://sonosim. ttlms.com/signon/). Teaching and examination are performed in groups of four to seven students per tutor. Multiple choice questions (MCQs) and OSCE stations are used for the examination.

\section{Copenhagen, Denmark}

\section{Local structure}

At the Medical University of Copenhagen, 546 students were accepted in 2018. The curriculum of medicine is scheduled for six years. Thereafter, one year of practical training as an intern is mandatory to obtain the right to practice independently as a physician.

\section{Content of lectures}

All students receive lectures about the basic physics of ultrasound. Ultrasound is included in the anatomy classes, in a specific one-day ultrasound course, in an imaging course, and in classes from other medical specialties as an adjunct. In the $6^{\text {th }}$ year, an optional eightweek course in ultrasound is offered that is designed so that students can obtain the level of expertise of a first-year resident in radiology (see below).

\section{Who is teaching?}

Radiologists, who are associate professors or professors at the university, are in charge of the lectures. Residents in radiology, specially trained medical students, associate professors and professors are involved in the hands-on training.

\section{Hands-on courses}

A full-day course is offered in ultrasound in the sixth semester (not mandatory), which includes four hours of hands-on practice. Furthermore, one hour is offered later in the semester. Hands-on is also included in the teaching from several other medical specialties without formal hands-on training. 


\section{Examinations (learning success, testing)}

A question regarding ultrasound may or may not be included in the OSCE exam in the $4^{\text {th }}$ year. For the optional eight-week course, a test on an abdominal ultrasound simulator (the Copenhagen Test) must be passed before beginning training in the radiology department. At the end of the course, students must pass a practical exam demonstrating abdominal pathology in a patient.

\section{London, UK}

\section{Local structure}

At Imperial College Medical School London, approximately 350 380 students are accepted each year. The whole curriculum requires six years, with the first three years of pre-clinical study that includes an eight-month period of research, which leads to a Bachelor of Science degree. The following three years are clinical years spent in teaching and district general hospitals, as well as within primary care, which leads to a Bachelor of Medicine and Bachelor of Surgery degree. This is then followed by a two-year internship before interns become fully registered doctors with the general medical council. The exposure to ultrasound varies among the 40 medical schools in the UK, but, in general, there is little exposure to ultrasound during the undergraduate years.

\section{Content of ultrasound lectures}

The teaching of anatomy now includes radiological anatomy in a clinical context. The lectures and teaching modules, however, usually comprise the use of cross-sectional modalities, namely, CT or MRI, which are easier to correlate with anatomical cross-sections. Ultrasound may be mentioned on occasion but does not feature heavily during the course at Imperial College London. The $5^{\text {th }}$ year students spend a week in radiology departments and are exposed to all modalities, including ultrasound. There are e-learning modules that have some ultrasound content, but these are taught more in terms of body parts and systems rather than being modality-based.

\section{Who is teaching?}

Radiologists and sonographers hold lectures on the physical background, as well as on ultrasound anatomy and pathology. During the clinical years, depending on the specialty, students may be taught some US by specialists in the particular field, especially if it is performed as part of point-of-care ultrasound e. g., in the emergency department and anesthesia department.

\section{Hands-on courses}

There is a "hands-on" US afternoon during the one-week secondment to radiology in year 5 where students get to scan each other, typically in groups of five to six, with a radiologist or sonographer as an instructor.

\section{Examinations (learning success, testing)}

Ultrasound knowledge is not formally examined but may be part of an anatomy question or year 3 exam, depending on what specialty is chosen for the student's research project. Ultrasound may also be part of an objective structured clinical examination (OSCE) in year 6 .

\section{Discussion}

Over the last several years, EFSUMB [1,2] and WFUMB [3] as well as several other publications have addressed the issue of student training in ultrasound [4-12] and promote ultrasound as an educational tool that should be used in the modern teaching of medicine.

Due to the fact that ultrasound allows real-time, noninvasive visualization of anatomic structures and their physiological function in vivo, ultrasound has been advocated as an auxiliary educational tool, in particular, for anatomy and physiology teaching ("living anatomy"), as well as for the teaching of physical examinations and basic clinical skills [13-21]. There is already consistent evidence that this type of teaching is also very well accepted, wanted, and enjoyed by students $[3,14,22]$, even if the evidence regarding the effect of US on external outcomes is still limited [14, 23, 24].

The present paper indicates that formal ultrasound education is beginning to enter the curricula of medical schools in Europe. While ultrasound training is already well established at some universities, responses from other universities indicate that ultrasound plays no or only a small role. Not surprisingly, most of the respondents felt that ultrasound knowledge is useful for all physicians. This high enthusiasm for ultrasound by the respondents is most likely biased to some degree, as the respondents belonged mainly to universities with already established US training programs or which were otherwise involved in US education.

Although the respondents acknowledged the importance of US education in medical universities, the mean hours of US training were reported to be as low as seven hours.

Interestingly, hands-on skills in ultrasound were taught in only $56 \%$ of the responding universities. One reason for the low proportion of hands-on training may be explained by the fact that the responses indicated that, although the benefits of the integration of ultrasound into the curricula are well understood, lack of time and limited faculty funding are perceived as major obstacles. Lack of time as well as a lack of funding are both related to the fact that hands-on ultrasound training requires small groups of students, which, in turn, requires a high number of teachers and equipment. Examples from Vienna and Ulm, as well as from other medical universities around the world, show that trained students can support staff physicians in curricular training, and thus, reduce personnel time and costs $[3,13,25,26]$. The added value and the teaching quality of student tutors in ultrasound education have not been well investigated scientifically thus far. However, as has been shown for other fields of medical education [27, 28], data suggest that results of peer-assisted ultrasound learning in undergraduate medical education may be equal to that of traditional faculty teaching [11,29-32]. Teach-the-teacher curricula should be developed, with the aim of optimizing student tutor-based training. An example of such a student tutor training curriculum is the "Young Sonographers" of the Swiss Society of Ultrasound in Medicine (SGUM), which was developed in cooperation with student groups and the Institute of Primary Health Care in Bern [3]. Other initiatives that used students as mentors in ultrasound have proved to be successful $[26,33,34]$, with the students acquiring adequate teaching skills in a relatively short training period, thus suggesting again that peer mentoring can facilitate the large-scale implementation of ultrasound teaching in undergraduate student education. 
The financial burdens and demand on teachers indicated as obstacles for the integration of ultrasound into the curricula can in part be overcome by implementing e-learning, as practiced in Gdansk, Timisoara and Uppsala [35, 36]. Other options are ultrasound phantoms and simulators which are implemented in several ultrasound teaching programs for medical students, for example, in Riga and Copenhagen. They may help to learn to practice medical ultrasound in a protected learning environment before practicing the technique in a clinical setting [37-41].

As the response rate of the survey was very low, the results do not give a comprehensive overview of ultrasound education in Europe. Presumably, the response rate was higher from universities in which ultrasound was integrated to some degree into the respective curricula. Furthermore, the selection of the universities to report on ultrasound activities was not representative but was mainly intended to give an overview of the different approaches from a variety of universities.

\section{Conclusion and Perspectives}

The present paper provides an overview of the recent early stage of activities and attempts of different European medical faculties to introduce sonography teaching into their curricula. Apparently, there is still a long way to go until diagnostic ultrasound will be reliably taught as a mandatory part of a medical school's or medical faculty's curriculum to all students. National and international medical ultrasound societies should, therefore, serve as platforms with which to coordinate and facilitate the process of forming effective medical student teaching methodology. Future EFSUMB initiatives will continue to promote the introduction of ultrasound as an integrative part of the core curriculum of student medical education and the preparation of proper teaching material.

\section{Conflict of Interest}

The authors declare that they have no conflict of interest.

\section{References}

[1] Cantisani V, Dietrich CF, Badea R, Dudea S, Prosch H, Cerezo E, Nuernberg $D$ et al. EFSUMB statement on medical student education in ultrasound [short version]. Ultraschall Med 2016; 37: 100-102

[2] Cantisani V, Dietrich CF, Badea R, Dudea S, Prosch H, Cerezo E, Nuernberg $D$ et al. EFSUMB Statement on Medical Student Education in Ultrasound [long version]. Ultrasound Int Open 2016; 2: E2-E7

[3] Dietrich CF, Hoffmann B, Abramowicz J, Badea R, Braden B, Cantisani V, Chammas MC et al. Medical Student Ultrasound Education: A WFUMB Position Paper, Part I. Ultrasound Med Biol 2019; 45: 271-281

[4] Dinh VA, Fu JY, Lu S, Chiem A, Fox JC, Blaivas M. Integration of Ultrasound in Medical Education at United States Medical Schools: A National Survey of Directors' Experiences. J Ultrasound Med 2016; 35 : 413-419

[5] Baltarowich OH, Di Salvo DN, Scoutt LM, Brown DL, Cox CW, DiPietro MA, Glazer DI et al. National ultrasound curriculum for medical students. Ultrasound Q 2014; 30: 13-19
[6] Dinh VA, Lakoff D, Hess ], Bahner DP, Hoppmann R, Blaivas M, Pellerito JS et al. Medical Student Core Clinical Ultrasound Milestones: A Consensus Among Directors in the United States. J Ultrasound Med 2016; 35: 421-434

[7] Chiem AT, Soucy Z, Dinh VA, Chilstrom M, Gharahbaghian L, Shah V, Medak $A$ et al. Integration of Ultrasound in Undergraduate Medical Education at the California Medical Schools: A Discussion of Common Challenges and Strategies From the UMeCali Experience. J Ultrasound Med 2016; 35: 221-233

[8] Heinzow HS, Friederichs H, Lenz P, Schmedt A, Becker JC, Hengst K, Marschall B et al. Teaching ultrasound in a curricular course according to certified EFSUMB standards during undergraduate medical education: a prospective study. BMC Med Educ 2013; 13: 84

[9] Wakefield RJ, Weerasinghe A, Tung P, Smith L, Pickering J, Msimanga T, Arora $\mathrm{M}$ et al. The development of a pragmatic, clinically driven ultrasound curriculum in a UK medical school. Med Teach 2018; 40: 600-606

[10] Bahner DP, Adkins EJ, Hughes D, Barrie M, Boulger CT, Royall NA. Integrated medical school ultrasound: Development of an ultrasound vertical curriculum. Crit Ultrasound J 2013; 5: 6

[11] Celebi N, Griewatz ], Malek NP, Krieg S, Kuehnl T, Muller R, Pauluschke-Frohlich J et al. Development and implementation of a comprehensive ultrasound curriculum for undergraduate medical students - a feasibility study. BMC Med Educ 2019; 19: 170

[12] Wilson SP, Mefford JM, Lahham S, Lotfipour S, Subeh M, Maldonado G, Spann $S$ et al. Implementation of a 4-Year Point-of-Care Ultrasound Curriculum in a Liaison Committee on Medical Education-Accredited US Medical School. J Ultrasound Med 2017; 36: 321-325

[13] Tarique U, Tang B, Singh M, Kulasegaram KM, Ailon J. Ultrasound Curricula in Undergraduate Medical Education: A Scoping Review. J Ultrasound Med 2018; 37: 69-82

[14] Davis J], Wessner CE, Potts ], Au AK, Pohl CA, Fields JM. Ultrasonography in Undergraduate Medical Education: A Systematic Review. J Ultrasound Med 2018; 37: 2667-2679

[15] Mircea PA, Badea R, Fodor D, Buzoianu AD. Using ultrasonography as a teaching support tool in undergraduate medical education - time to reach a decision. Med Ultrason 2012; 14: 211-216

[16] So S, Patel RM, Orebaugh SL. Ultrasound imaging in medical student education: Impact on learning anatomy and physical diagnosis. Anat, Sci Educ 2017; 10: 176-189

[17] Patel SG, Benninger B, Mirjalili SA. Integrating ultrasound into modern medical curricula. Clin Anat 2017; 30: 452-460

[18] Walrod BJ, Schroeder A, Conroy M], Boucher LC, Bockbrader M, Way $\mathrm{DP}, \mathrm{McC}$ amey KL et al. Does Ultrasound-Enhanced Instruction of Musculoskeletal Anatomy Improve Physical Examination Skills of First-Year Medical Students? J Ultrasound Med 2018; 37: 225-232

[19] Luetmer MT, Cloud BA, Youdas JW, Pawlina W, Lachman N. Simulating the multi-disciplinary care team approach: Enhancing student understanding of anatomy through an ultrasound-anchored interprofessional session. Anat Sci Educ 2018; 11: 94-99

[20] Danielle F. Royer, Seeing with Sound: How Ultrasound Is Changing the Way We Look at Anatomy. In: Rea PM, Ed. Biomedical Visualisation, Vol. 2. Cham: Springer Nature Switzerland; 2019: 1-11

[21] Dinh VA, Frederick J, Bartos R, Shankel TM, Werner L. Effects of ultrasound implementation on physical examination learning and teaching during the first year of medical education. J Ultrasound Med 2015; 34: 43-50

[22] Smith JP, Kendall JL, Royer DF. Improved medical student perception of ultrasound using a paired anatomy teaching assistant and clinician teaching model. Anat Sci Educ 2018; 11: 175-184 
[23] Feilchenfeld Z, Dornan T, Whitehead C, Kuper A. Ultrasound in undergraduate medical education: A systematic and critical review. Med Educ 2017; 51: 366-378

[24] Jamniczky HA, Cotton D, Paget M, Ramji Q, Lenz R, McLaughlin K, Coderre $S$ et al. Cognitive load imposed by ultrasound-facilitated teaching does not adversely affect gross anatomy learning outcomes. Anat Sci Educ 2017; 10: 144-151

[25] Hamza A, Radosa JC, Solomayer EF, Takacs Z, Juhasz-Boess I, Stroder R, Joukhadar R et al. Introduction of a student tutor-based basic obstetrical ultrasound screening in undergraduate medical education. Arch Gynecol Obstet 2019; 300: 59-66

[26] Ahn JS, French AJ, Thiessen ME, Kendall JL. Training peer instructors for a combined ultrasound/physical exam curriculum. Teach Learn Med 2014; 26: 292-295

[27] Rees EL, Quinn P], Davies B, Fotheringham V. How does peer teaching compare to faculty teaching? A systematic review and meta-analysis (.). Med Teach 2016; 38: 829-837

[28] Burgess A, McGregor D, Mellis C. Medical students as peer tutors: A systematic review. BMC Med Educ 2014; 14: 115

[29] Knobe M, Munker R, Sellei RM, Holschen M, Mooij SC, Schmidt-Rohlfing B, Niethard FU et al. Peer teaching: A randomised controlled trial using student-teachers to teach musculoskeletal ultrasound. Med Educ 2010; 44: 148-155

[30] Celebi N, Zwirner K, Lischner U, Bauder M, Ditthard K, Schurger S, Riessen $R$ et al. Student tutors are able to teach basic sonographic anatomy effectively - a prospective randomized controlled trial. Ultraschall Med 2012; 33: 141-145

[31] Byrne C, Kahl N, Knight B, Lee M, Morley S, Lahham S, Bingisser R et al. A prospective evaluation of point of care ultrasound teaching in Switzerland. J Med Ultrasound 2019; 27: 92-96

[32] Kuhl M, Wagner R, Bauder M, Fenik Y, Riessen R, Lammerding-Koppel M, Gawaz $\mathrm{M}$ et al. Student tutors for hands-on training in focused emergency echocardiography - a randomized controlled trial. BMC Med Educ 2012; 12: 101
[33] Garcia-Casasola G, Sanchez F], Luordo D, Zapata DF, Frias MC, Garrido VV, Martinez JV et al. Basic abdominal point-of-care ultrasound training in the undergraduate: Students as mentors. J Ultrasound Med 2016; 35: 2483-2489

[34] Celebi N, Griewatz J, Malek NP, Hoffmann T, Walter C, Muller R, Riessen R et al. Outcomes of three different ways to train medical students as ultrasound tutors. BMC Med Educ 2019; 19: 125

[35] Hempel D, Haunhorst S, Sinnathurai S, Seibel A, Recker F, Heringer F, Michels $G$ et al. Social media to supplement point-of-care ultrasound courses: The "sandwich e-learning" approach. A randomized trial. Crit Ultrasound | 2016; 8: 3

[36] Hempel D, Sinnathurai S, Haunhorst S, Seibel A, Michels G, Heringer F, Recker $\mathrm{F}$ et al. Influence of case-based e-learning on students' performance in point-of-care ultrasound courses: A randomized trial. Eur J Emerg Med 2016; 23: 298-304

[37] Ostergaard ML, Ewertsen C, Konge L, Albrecht-Beste E, Bachmann Nielsen M. Simulation-based abdominal ultrasound training - A systematic review. Ultraschall Med 2016; 37: 253-261

[38] Miller GT, Scerbo MW, Zybak S, Byars DV, Goodmurphy CW, Lattanzio FA, Knapp BJ et al. Learner improvement from a simulation-enhanced ultrasonography curriculum for first-year medical students. J Ultrasound Med 2017; 36: 609-619

[39] Shah S, Tohmasi S, Frisch E, Anderson A, Almog R, Lahham S, Bingisser $R$ et al. A comparison of simulation versus didactics for teaching ultrasound to Swiss medical students. World J Emerg Med 2019; 10: 169-176

[40] Orr KE, Hamilton SC, Clarke R, Adi MY, Gutteridge C, Suresh P, Freeman SJ. The integration of transabdominal ultrasound simulators into an ultrasound curriculum. Ultrasound 2019; 27: 20-30

[41] Ostergaard ML, Rue Nielsen K, Albrecht-Beste E, Kjaer Ersboll A, Konge L, Bachmann Nielsen M. Simulator training improves ultrasound scanning performance on patients: a randomized controlled trial. Eur Radiol 2019; 29 : $3210-3218$ 